H.-J. Choo - J.-H. Kim • O.-B. Kwon - C. S. Lee •

J. Y. Mun · S. S. Han - Y.-S. Yoon - G. Yoon ·

K.-M. Choi · Y.-G. Ko

\title{
Mitochondria are impaired in the adipocytes of type 2 diabetic mice
}

Received: 3 September 2005 / Accepted: 27 November 2005 / Published online: 25 February 2006

C) Springer-Verlag 2006

\begin{abstract}
Aims/hypothesis: The aim of this study was to confirm a link between mitochondrial dysfunction and type 2 diabetes. Materials and methods: Cellular levels of mitochondrial proteins, cellular mitochondrial DNA content, and mitochondrial function and morphology were assessed by MitoTracker staining and electron microscopy, in white adipose tissue of 12-week-old male wild-type, obese $(o b / o b)$, and diabetic $(d b / d b)$ mice. Results: Levels of mitochondrial proteins were found to be very similar in the livers and muscles of all the mice studied. However, levels were greatly decreased in the adipocytes of $d b / d b$ mice, but not in those of the wild-type and $o b / o b$ mice. Levels of mitochondrial DNA were also found to be considerably reduced in the adipocytes of $d b / d b$ mice. MitoTracker staining and under electron microscopy revealed that the number of mitochondria was reduced in adipocytes of $d b / d b$ mice. Respiration and fatty acid oxidation studies indicated mitochondrial dysfunction in adipocytes of $d b / d b$ mice. Interestingly, there was an increase in mitochondria and mitochondrial protein production in adipocytes of $d b / d b$ mice treated with rosiglitazone, an agent that enhances insulin sensitivity. Conclusions/interpretation: Taken together, these data
\end{abstract}

Electronic Supplementary Material Supplementary material is available for this article at http://dx.doi.org/10.1007/s00125-0060170-2

H.-J. Choo · J.-H. Kim · O.-B. Kwon · C. S. Lee · J. Y. Mun

S. S. Han · Y.-G. Ko $(\bowtie)$

Graduate School of Life Sciences and Biotechnology,

Korea University,

1, 5-ka, Anam-dong,

Sungbuk-gu, Seoul, 136-701, South Korea

e-mail: ygko@korea.ac.kr

Tel.: +82-2-32903453

Fax: +82-2-9279028

Y.-S. Yoon · G. Yoon

College of Medicine, Ajou University,

Suwon, Kyunggi-Do, South Korea

K.-M. Choi

Guro Hospital, College of Medicine, Korea University,

Seoul, South Korea indicate that mitochondrial loss in adipose tissue is correlated with the development of type 2 diabetes.

Keywords Adipocyte $\cdot$ Diabetes $\cdot$ Mitochondria Rosiglitazone

Abbreviations AMPK: AMP-activated protein kinase ESM: Electronic Supplementary Material - LETO: LongEvans Tokushima Otsuka $\cdot$ mtDNA: mitochondrial DNA OLETF: Otsuka Long-Evans Tokushima fatty - OxPhos: oxidative phosphorylation complex $\cdot \operatorname{PGC} 1 \alpha$ : PPAR $\gamma$ coactivator $1 \alpha \cdot \operatorname{PPAR} \gamma$ : peroxisome proliferator-activated receptor $\gamma$

\section{Introduction}

Type 2 insulin-resistant diabetes mellitus is prevalent in modern society due to ample food supplies coupled with sedentary lifestyles. Liver, muscle and adipose tissue are resistant to insulin action in type 2 diabetes, and this can ultimately result in several serious complications, including kidney failure, blindness, damage to the feet and legs (which can consequently require amputation), and a much increased risk of heart attack and stroke $[1,2]$. Moreover, a link between type 2 diabetes and an increased incidence of Alzheimer's disease has been established [3].

Mitochondrial dysfunction has been implicated in the development of type 2 diabetes $[4,5]$. For example, skeletal muscle of patients with type 2 diabetes exhibits reduced mitochondrial oxidative activity, as well as decreases in the level of ATP synthase $\beta$, an essential protein for mitochondrial respiration [6]. Indeed, intramyocellular mitochondria tend to be smaller, and subsarcolemmal mitochondria are more rarely found in the skeletal muscle of type 2 diabetic patients than in muscle obtained from lean volunteers $[7,8]$. Thus, skeletal muscle of patients with type 2 diabetes exhibits reduced fatty acid oxidation activity and a high intramyocellular lipid content $[9,10]$.

Adipose tissue is not only an energy reservoir of lipid droplets composed of triglycerides and cholesterol, but is 
also an important organ in the maintenance of blood glucose homeostasis. For example, the adipose-selective depletion of the gene encoding GLUT4 in mice leads to impaired glucose tolerance, insulin resistance and hyperinsulinaemia [11]. In addition to its function as an energy reservoir, white adipose tissue functions as an endocrine organ, secreting a host of different cytokines [12]. Thus, insulin resistance may be the result of increased visceral adiposity and abnormal levels of adipocyte cytokines such as leptin, resistin, adiponectin and TNF [13]. Adipocytederived leptin regulates food intake and improves insulin sensitivity [14]. Adiponectin increases fatty acid combustion via AMP-activated protein kinase (AMPK) activation in muscle cells, whereas resistin and TNF reduce sensitivity to insulin [15-17]. Some patients with type 2 diabetes exhibit low levels of adiponectin and high levels of resistin and TNF in their blood [13], implying that these cytokines play important roles in the development of the disease.

As the mitochondria in white adipose tissue are involved in lipid metabolism, including lipogenesis and lipolysis, adipogenesis is accompanied by mitochondrial biogenesis. Indeed, mitochondria are generated at very high levels when preadipocyte 3T3-L1 cells are differentiated into adipocytes [18, 19]. More interestingly, mitochondrial biogenesis and activity are significantly increased when adipocytes are treated with rosiglitazone, a peroxisome proliferator-activated receptor $\gamma(\operatorname{PPAR} \gamma)$ agonist that modulates the differentiation and function of adipocytes [20]. As PPAR $\gamma$ agonists alleviate insulin resistance and lower glucose levels in type 2 diabetic rodents and patients [12], it is tempting to speculate that PPAR $\gamma$ agonists exert an effect in type 2 diabetes, as they induce mitochondrial biogenesis in adipocytes.

Mitochondrial dysfunction in liver, muscle and adipose tissue has not been analysed in type 2 diabetic animals, even though it is known that these organs are major targets in type 2 diabetes. In order to confirm the connection between mitochondrial dysfunction and type 2 diabetes, we attempted to assess mitochondrial biogenesis in liver, skeletal muscle and adipose tissue isolated from normal, obese $(o b / o b)$ and diabetic $(d b / d b)$ mice.

\section{Materials and methods}

Animals and antibodies Diabetic $d b / d b$ mice and their lean littermates $(\mathrm{db} /+$ and $\mathrm{C} 57 \mathrm{BL} / \mathrm{KSJ})$ resultant from breeding with male and female $d b /+$ mice (breeding pair), and $o b /$ $o b$ mice and their lean littermates $(o b /+$ and $\mathrm{C} 57 \mathrm{BL} / 6 \mathrm{~J})$ resultant from breeding with male and female $o b /+$ mice (breeding pair) (Jackson Laboratories, Bar Harbor, ME, USA), were housed in plastic cages on a $12: 12 \mathrm{~h}$ lightdark photocycle with free access to water and food (Purina 5008; Ralston-Purina, St Louis, MO, USA), and were studied at 12 weeks of age. Four-week-old Long-Evans Tokushima Otsuka (LETO) rats and Otsuka Long-Evans Tokushima fatty (OLETF) rats were donated by Otuska Pharmaceuticals [21], and were housed in plastic cages on a 12:12 h light-dark photoperiod with free access to water and food, and were studied at 33 weeks of age. Animals were handled according to the Principles of Laboratory Animal Care (NIH Publication no. 85-23, revised 1985).

Antibodies directed against ATP synthase $\alpha$ and $\beta$, oxidative phosphorylation complex (OxPhos) II (succinate:ubiquinone oxidoreductase $70 \mathrm{kDa}$ subunit), and OxPhos III (ubiquinone:cytochrome c oxidoreductase core I subunit) were obtained from Molecular Probes (Eugene, OR, USA). The anti-clathrin heavy chain antibodies used were obtained from BD Biosciences (North Ryde, NSW, Australia) and anti- $\beta$-actin antibodies were obtained from Santa Cruz Biotechnology (Santa Cruz, CA, USA).

Adipocyte isolation Dissected epididymal fat pads from mice were immersed in Krebs Ringer HEPES buffer, containing $120 \mathrm{mmol} / 1 \mathrm{NaCl}, 4 \mathrm{mmol} / 1 \quad \mathrm{KH}_{2} \mathrm{PO}_{4}$, $17 \mathrm{mmol} / 1 \mathrm{MgSO}_{4} \cdot 7 \mathrm{H}_{2} \mathrm{O}, 1 \mathrm{mmol} / 1 \mathrm{CaCl}_{2}, 10 \mathrm{mmol} / \mathrm{l}$ $\mathrm{NaHCO}_{3}, 30 \mathrm{mmol} / \mathrm{l}$ HEPES, $2.5 \mathrm{mmol} / \mathrm{l}$ D-glucose, $200 \mathrm{nmol} / 1$ adenosine, $2 \%$ albumin, adjusted to $\mathrm{pH} 7.4$. Adipose tissues were minced and digested with $2.5 \mathrm{mg} / \mathrm{ml}$ collagenase (Sigma-Aldrich, St Louis, MO, USA) for $1 \mathrm{~h}$ at $37^{\circ} \mathrm{C}$, in a shaking water-bath. The suspension was centrifuged, and the floating adipocytes were collected and filtered through $400-\mu \mathrm{m}$ nylon mesh to remove tissue pieces. The cell suspension was then centrifuged at $400 \mathrm{~g}$ for $1 \mathrm{~min}$. Adipocytes were collected from the supernatants and then resuspended in cold PBS. Centrifugation was repeated three times.

Immunoblotting Liver and muscle were lysed with RIPA lysis buffer $(25 \mathrm{mmol} / 1$ Tris- $\mathrm{HCl}, \mathrm{pH} 7.4,150 \mathrm{mmol} / \mathrm{l}$ $\mathrm{NaCl}, 1 \%$ Triton X-100, $1 \%$ sodium deoxycholate, $0.1 \%$ SDS, $2 \mathrm{mmol} / 1 \mathrm{EDTA}, 50 \mathrm{mmol} / \mathrm{l}$ sodium fluoride, $0.2 \mathrm{mmol} / 1$ sodium vanadate, $1 \mathrm{mmol} / 1$ phenylmethylsulfonylfluoride (PMSF) and protease inhibitor cocktail [Roche Applied Science, Mannheim, Germany]) and adipocytes were lysed with $1 \%$ sodium deoxycholate, $1 \%$ Triton X-100 and protease inhibitor cocktail (Roche Applied Science). After $10 \mathrm{~min}$ of microcentrifugation at $20,000 \mathrm{~g}$ and $4^{\circ} \mathrm{C}$, the whole-cell lysates (supernatant) were separated by SDS-PAGE, and then transferred to nitrocellulose membranes. Antigens were visualised by sequential treatment with specific antibodies, horseradish peroxidase-conjugated secondary antibodies and an enhanced chemiluminescence substrate kit (Intron, Seoul, South Korea).

Mitochondrial DNA analysis To determine the intracellular mitochondrial DNA (mtDNA) levels, two primer sets-a set for ND2 (which encodes subunit 2 of OxPhos I [22]), found in mtDNA, and a set for 28s RNA (also known as LOC236598, which encodes 28S rRNA), found in nuclear DNA - were used for PCR analysis, with the isolated total genomic DNA used as a template. The final PCR products were electrophoresed on a $1.5 \%$ agarose gel, stained with $0.5 \mu \mathrm{g} / \mathrm{ml}$ of ethidium bromide solution, and visualised with a UV transilluminator (Gel Doc 2000; Bio-Rad, Milan, Italy). The ratio of $N D 2: 28 s R N A$ was determined by scanning and comparing the intensities, and was expressed 
as a percentage of the control. The sequences of the primer sets used in this study were as follows: for mitochondrial ND2, 5'-CAAGGGATCCCACTGCACATAGG-3'

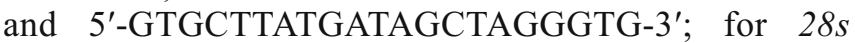
$R N A, 5^{\prime}$-TAGCAGCCGACTTAGAACTGG-3' and 5'CTCCCACTTATT-CTACACCTC-3'.

MitoTracker staining of adipose tissue Adipose tissues of $d b / d b, o b / o b$ and wild-type mice were fixed with $4 \%$ paraformaldehyde and embedded in paraffin wax. Paraffin sections (4-6 $\mu \mathrm{m}$ thick) were dewaxed in xylene and rehydrated through graded ethanol. For mitochondria staining, sections were incubated with $250 \mathrm{nmol} / 1$ MitoTracker (Molecular Probes) for $1 \mathrm{~h}$ at room temperature. Slides were washed and had coverslips mounted, and were then observed with a Bio-Rad confocal microscope (MRC 1024; Bio-Rad, Hercules, CA, USA).

Electron microscopy For ultrastructural examinations, liver, muscle and adipocytes were fixed for $1.5-2 \mathrm{~h}$ at $4^{\circ} \mathrm{C}$ in $0.1 \mathrm{~mol} / 1$ sodium phosphate buffer $(\mathrm{pH} 7.4)$ containing a $2 \%$ paraformaldehyde, $2.5 \%$ glutaraldehyde mixture. The tissues were post-fixed for $2 \mathrm{~h}$ in the same buffer containing $1 \% \mathrm{OsO}_{4}$. The tissue pieces were subsequently washed and dehydrated using graded acetone, and embedded in Epon-Araldite. Thick and thin sections were prepared on an ultra microtome (MT-X; RMC, Tucson, AZ, USA). The sectioned thick tissues were then stained with $1 \%$ toluidine blue borax solution, mounted on copper grids, and double-stained with uranyl acetate. The grids were examined in a JEM 100 CX-II electron microscope (JEOL, Tokyo, Japan).

Fatty acid oxidation assay This method has previously been described in detail [20]. Briefly, isolated adipocytes were washed in Krebs Ringer HEPES buffer plus 1\% BSA buffer. $\left[{ }^{14} \mathrm{C}\right]$ Palmitic acid (Perkin Elmer Life Sciences, Boston, MA, USA) was added at a final concentration of $7.4 \mathrm{KBq} / \mathrm{ml}$. An uncapped Eppendorf tube containing a piece of No. 1 Whatman filter paper soaked in $300 \mu \mathrm{l}$ benzethonium hydroxide (Sigma-Aldrich) was placed inside each $50-\mathrm{ml}$ tube. Tubes were then sealed and incubated at $37^{\circ} \mathrm{C}$ for $2 \mathrm{~h}$. Tubes were gently shaken every $30 \mathrm{~min}$ during the incubation. After $2 \mathrm{~h}, 100 \mu \mathrm{l}$ of $12 \mathrm{~mol} / 1$ $\mathrm{HCl}$ was added to the cells to release the ${ }^{14} \mathrm{CO}_{2}$, and each tube was resealed and incubated at $37^{\circ} \mathrm{C}$ overnight. The ${ }^{14} \mathrm{CO}_{2}$ released was measured by scintillation counting of the filter paper.

Oxygen consumption The protocol used has previously been described in detail [20]. Briefly, isolated adipocytes were divided into aliquots in a BD Oxygen Biosensor System plate (BD Biosciences, San Jose, CA, USA) in triplicate. Plates were sealed and read on a GENios Pro microplate spectrophotometer (Tecan, San Jose, CA, USA) at 1-min intervals for $30 \mathrm{~min}$ at an excitation wavelength of $485 \mathrm{~nm}$ and an emission wavelength of $612 \mathrm{~nm}$.
Statistical analysis Results were compared by ANOVA. A $p$ value of less than 0.05 was considered to be statistically significant.

\section{Results}

Mitochondrial biogenesis is reduced in the adipocytes of $\mathrm{db} / \mathrm{db}$ mice To investigate the association between type 2 diabetes and mitochondrial dysfunction, we investigated the expression levels of mitochondrial proteins from the muscle, liver, and adipose tissue of normal, obese $(o b / o b)$ and diabetic $(d b / d b)$ mice. Muscle, liver and adipocytes were isolated from 12-week-old $d b / d b, d b /+$ and $+/+$ (C57BL/KSJ) mice. Adipocytes were isolated from the epididymal fat pads, and muscle tissue was obtained from the femoral muscle. Proteins from each organ were then analysed by immunoblotting with anti-ATP synthase $\alpha$ and $\beta$ antibodies. As shown in Fig. 1a, and Electronic Supplementary Material (ESM) Table 1, no differences were detected in the levels of expression of ATP synthase $\alpha$ or $\beta$ in the muscle or liver of any of the mice. However, adipocytes from $d b / d b$ mice exhibited low levels of expression of mitochondrial proteins, including ATP synthase $\alpha$ and $\beta$, OxPhos II and OxPhos III (ubiquinone:cytochrome $\mathrm{c}$ oxidoreductase core I subunit). This indicates that in $d b / d b$ mice the mitochondria are severely and significantly diminished in number.

As this low expression of mitochondrial proteins in the adipocytes of $d b / d b$ mice may have occurred as a result of the obesity of these mice, we assessed mitochondrial protein expression levels in muscle, liver and adipocytes isolated from 12-week-old ob/ob, ob/t and +/+ $(\mathrm{C} 57 \mathrm{BL} / 6 \mathrm{~J})$ mice. As shown in Fig. $1 \mathrm{~b}$ and ESM Table 1, there were no differences between the groups of mice in terms of the expression of mitochondrial proteins in any of the organs isolated, suggesting that, in contrast with the $d b / d b$ mice, the $o b / o b$ mice do not exhibit severe mitochondrial loss.

To extend the findings on mitochondrial loss in the $d b / d b$ adipocytes, we next assessed the expression of mitochondrial proteins in the liver, muscle and adipocytes of OLETF rats, another animal model of type 2 diabetes. As expected, we failed to detect any differences between the control LETO and the OLETF rats in terms of the level of expression of ATP synthase $\alpha$ or $\beta$ in the liver or muscle (data not shown). However, the OLETF adipocytes were shown to express lower levels of mitochondrial proteins, such as ATP synthase $\alpha$ and $\beta$ and OxPhos II (succinate: ubiquinone oxidoreductase $70 \mathrm{kDa}$ subunit), than the control LETO adipocytes (Fig. 1c), thus suggesting that the OLETF adipocytes are characterised by mitochondrial dysfunction.

In order to confirm this mitochondrial loss in the adipocytes from $d b / d b$ mice, we measured levels of mitochondrial and genomic DNA in adipocytes isolated from 12-week-old $d b / d b, d b /+$ and $+/+$ mice. Rotenonesensitive NADH:ubiquinone oxidoreductase (OxPhos I, 
a

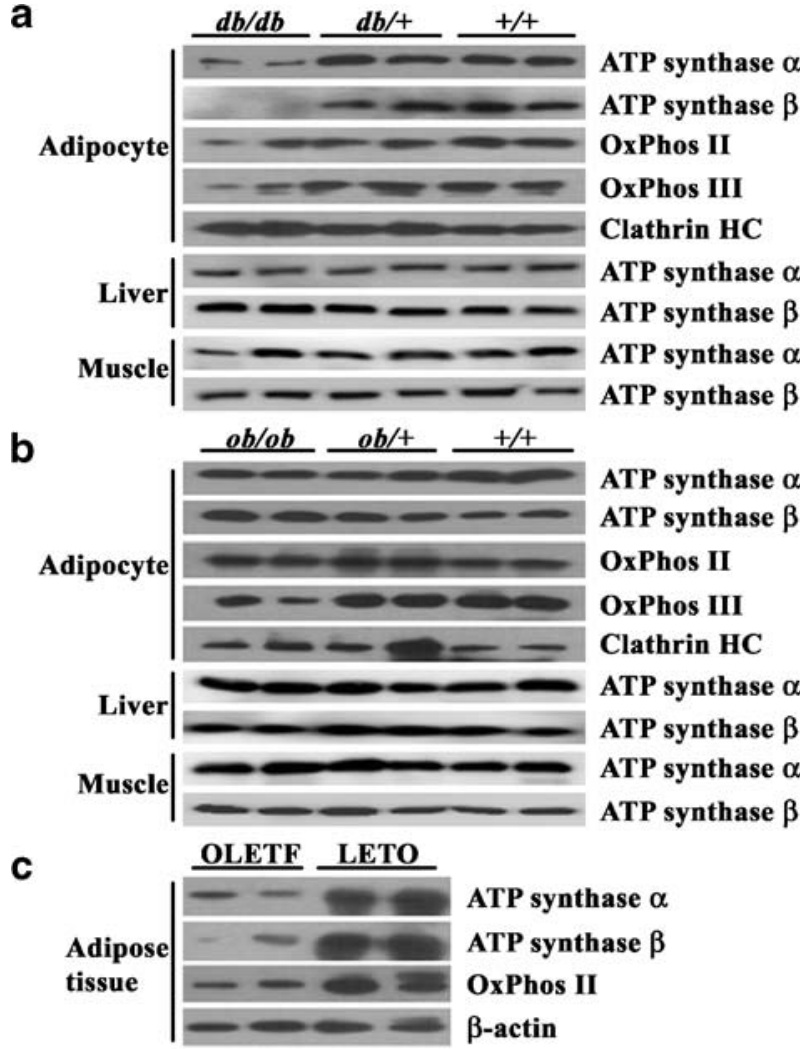

Fig. 1a,b Low expression levels of mitochondrial proteins in the adipocytes of $d b / d b$ mice. Adipocytes were isolated from epididymal adipose tissue of 12 -week-old male $d b / d b, d b /+$ and $+/+$ (C57BL/KSJ) mice (a), and 12-week-old male $o b / o b, o b /+$ and $+/+$ $(\mathrm{C} 57 \mathrm{BL} / 6 \mathrm{~J})$ mice $(\mathbf{b})$. Proteins $(30 \mu \mathrm{g})$ were extracted from the liver, femoral skeletal muscle and adipose tissue, and were subsequently analysed by immunoblotting with anti-ATP synthase $\alpha$ and $\beta$, antiOxPhos II (succinate:ubiquinone oxidoreductase $70 \mathrm{kDa}$ subunit), anti-OxPhos III (ubiquinone:cytochrome c oxidoreductase core I subunit) and anti-clathrin heavy chain (Clathrin HC) antibodies. The liver and skeletal muscle from five mice were analysed, and the adipocytes from 17 mice were analysed. Among the $17 \mathrm{db} / \mathrm{db}$ mice, the adipocytes of 15 mice exhibited low levels of ATP synthase $\alpha$ and $\beta$ expression, but those from the other two mice exhibited normal levels of expression. Two representative immunoblots are shown for each group of mice. c Adipose tissue from LETO and OLETF rats was lysed, and then analysed by immunoblotting with anti-ATP synthase $\alpha$ and $\beta$, anti-OxPhos II (succinate:ubiquinone oxidoreductase $70 \mathrm{kDa}$ subunit) and anti- $\beta$-actin antibodies

EC 1.6.5.3), a major complex of the mitochondrial respiratory chain, consists of at least 35 subunits of dual genetic origin: seven subunits (the NADH dehydrogenase subunits) are encoded by mtDNA, whereas the remainder are nuclear gene products. Thus, we were able to measure the mtDNA content of the cells by PCR using two primers designed to target $N D 2$, a mitochondrial gene. As shown in Fig. 2a, the adipocytes from the $d b / d b$ mice had a lower mtDNA content than those from the $d b /+$ and $+/+$ mice. When we measured the genomic DNA content of the adipocytes by PCR using two primers specifically designed to target the gene $28 s R N A$, we found this to be similar for all mice. The mtDNA content was also found to be reduced in the $o b / o b$ adipocytes (Fig. 2b). The ratio of mtDNA: genomic DNA in the $d b / d b$ and $o b / o b$ adipocytes was 0.7 and 1.1, respectively, whereas the ratio in the heterozygotic and wild-type adipocytes was 1.3 . This finding suggests that the $d b / d b$ and $o b / o b$ adipocytes exhibit a marked mitochondrial loss.

To provide further evidence of the mitochondrial loss from $d b / d b$ adipocytes, we determined the mitochondrial content of the adipocytes by staining with MitoTracker Red, a mitochondrion-specific dye. As shown in Fig. 3, $d b /$ $d b$ adipocytes were weakly stained with MitoTracker compared with adipocytes isolated from other mice, indicating that the adipocytes from $d b / d b$ mice contained fewer mitochondria than the adipocytes from other mice.

Abnormal mitochondrial morphologies from the adipocytes of $\mathrm{db} / \mathrm{db}$ and $\mathrm{ob} / \mathrm{ob}$ mice We investigated the morphology of the mitochondria from $d b / d b$ and $o b / o b$ adipocytes using electron microscopy. Adipocytes were isolated from the epididymal fat pads of 12-week-old $d b$ / $d b, d b /+$ and $+/+$ mice, and were visualised by electron microscopy. As shown in Fig. 4a,c,e, the mitochondria were almost completely absent from the adipocytes from the $d b / d b$ mice, but were present in the adipocytes from the $d b /+$ and $+/+$ mice, thereby re-confirming the

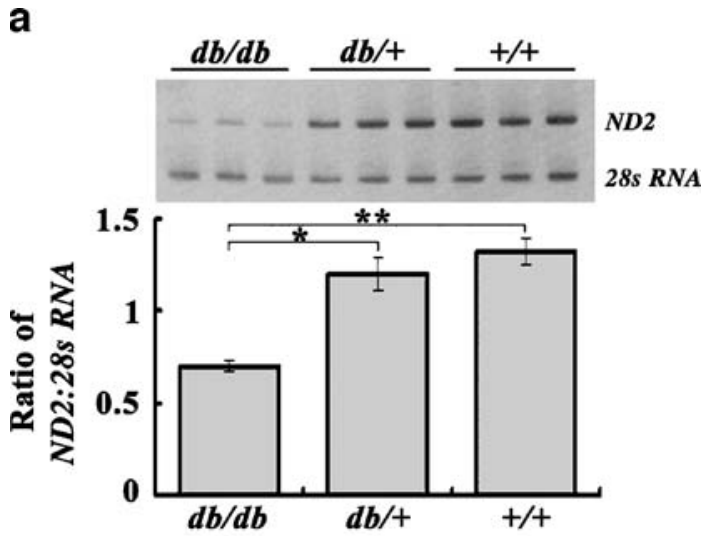

b

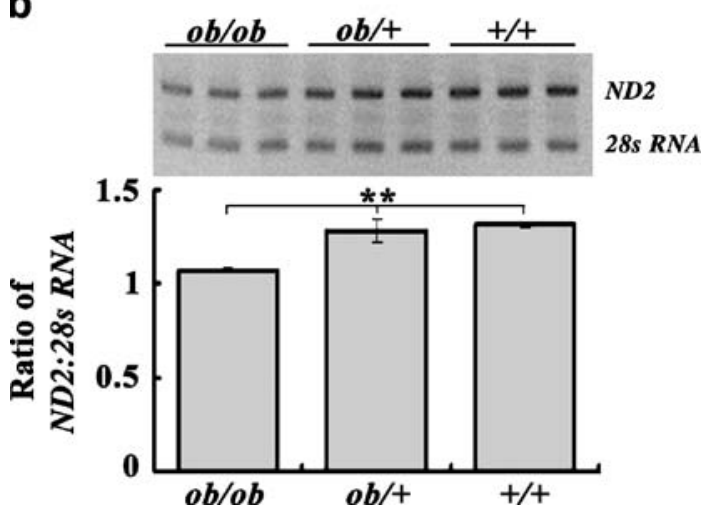

Fig. 2 Cellular mtDNA content is reduced in adipocytes of $d b / d b$ mice. Adipocytes were isolated from the epididymal adipose tissue of 12-week-old male $d b / d b, d b /+$ and $+/+(\mathrm{C} 57 \mathrm{BL} / \mathrm{KSJ})$ mice, and $o b / o b, o b /+$ and $+/+(\mathrm{C} 57 \mathrm{BL} / 6 \mathrm{~J})$ mice. The genomic DNA and mtDNA content of the adipocytes $(n=3)$ was assessed by PCR analysis with primers designed to target $28 s R N A$ and ND2 (which encodes subunit 2 of OxPhos I). The ratio of $N D 2: 28 s R N A$ was obtained by measuring the intensity of each band. ${ }^{*} p<0.05$; $* * p<0.01$ 

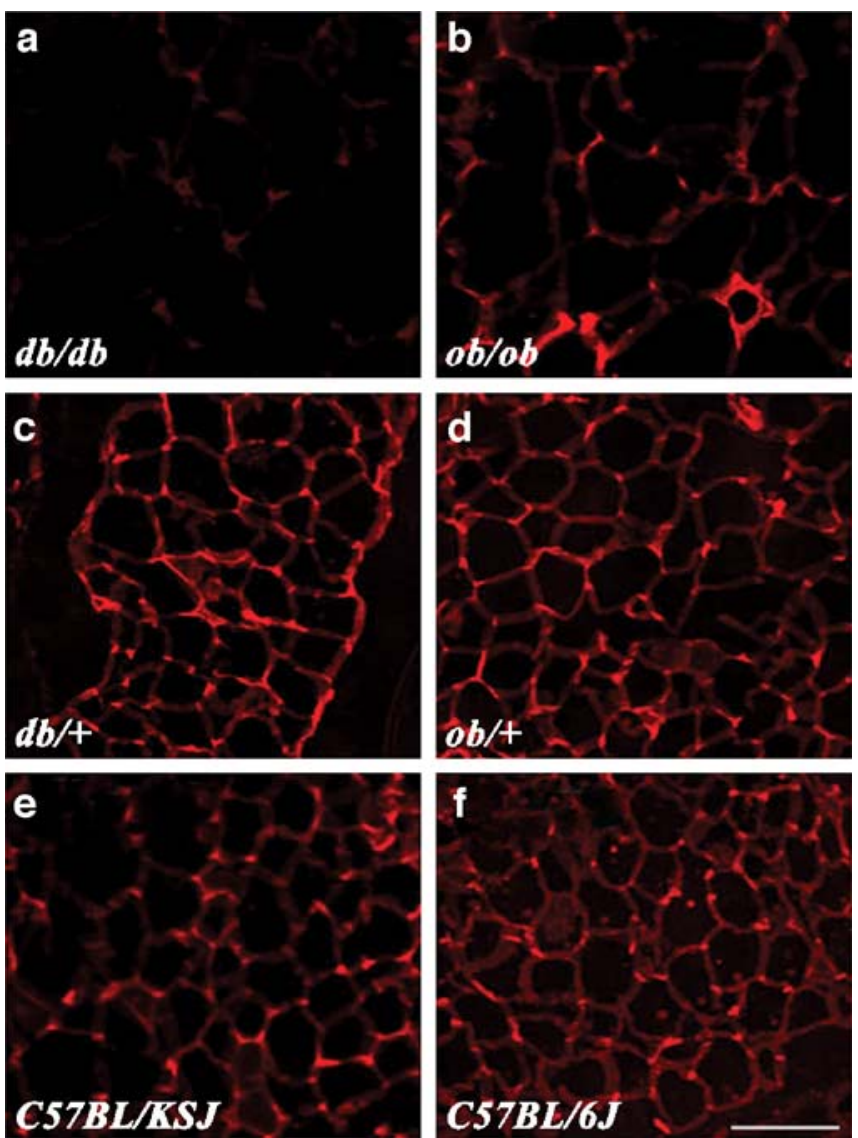

Fig. 3 Adipocytes from $d b / d b$ mice contain fewer mitochondria, as indicated by MitoTracker staining. Epididymal adipose tissue was isolated from 12-week-old male $d b / d b(\mathbf{a}), d b /+(\mathbf{c})$ and $+/+(\mathrm{C} 57 \mathrm{BL} /$ $\mathrm{KSJ})(\mathbf{e})$, and $o b / o b$ (b), $o b /+$ (d) and +/+ (C57BL/6J) (f) mice. Adipose tissue samples were fixed, sliced and then stained with MitoTracker Red 580. The white bar indicates $100 \mu \mathrm{m}$

mitochondrial loss in the $d b / d b$ adipocytes. Many mitochondria were observed in the adipocytes from the $o b / o b$ mice, but their morphology was quite different from that of the mitochondria from $o b /+$ and $+/+$ mice (Fig. $4 \mathrm{~b}, \mathrm{~d}, \mathrm{f}$ ). The mitochondria were smaller in the $o b / o b$ adipocytes than in the $o b /+$ and $+/+$ adipocytes, suggesting some mitochondrial dysfunction in the adipocytes from the $d b /$ $d b$ mice, as well as in those from the $o b / o b$ mice. We also observed mitochondrial morphology in skeletal muscle and liver from normal, $o b / o b$ and $d b / d b$ mice under electron microscopy. We found no differences between the groups of mice in terms of mitochondrial number or morphology (ESM Fig. 1).

Mitochondrial dysfunction of the adipocytes from diabetic mice To test the function of the mitochondria, we measured the rate of fatty acid oxidation in adipocytes from normal, obese $(o b / o b)$ and diabetic $(d b / d b)$ mice at 12 weeks of age. As shown in Fig. 5a, the adipocytes from $d b / d b$ mice showed lower levels of palmitic acid oxidation than the adipocytes from wild-type mice (C57BL/KSJ). In addition, adipocytes from $o b / o b$ mice also showed reduced fatty acid oxidation compared with adipocytes from C57BL/6J mice (Fig. 5a). In order to further demonstrate
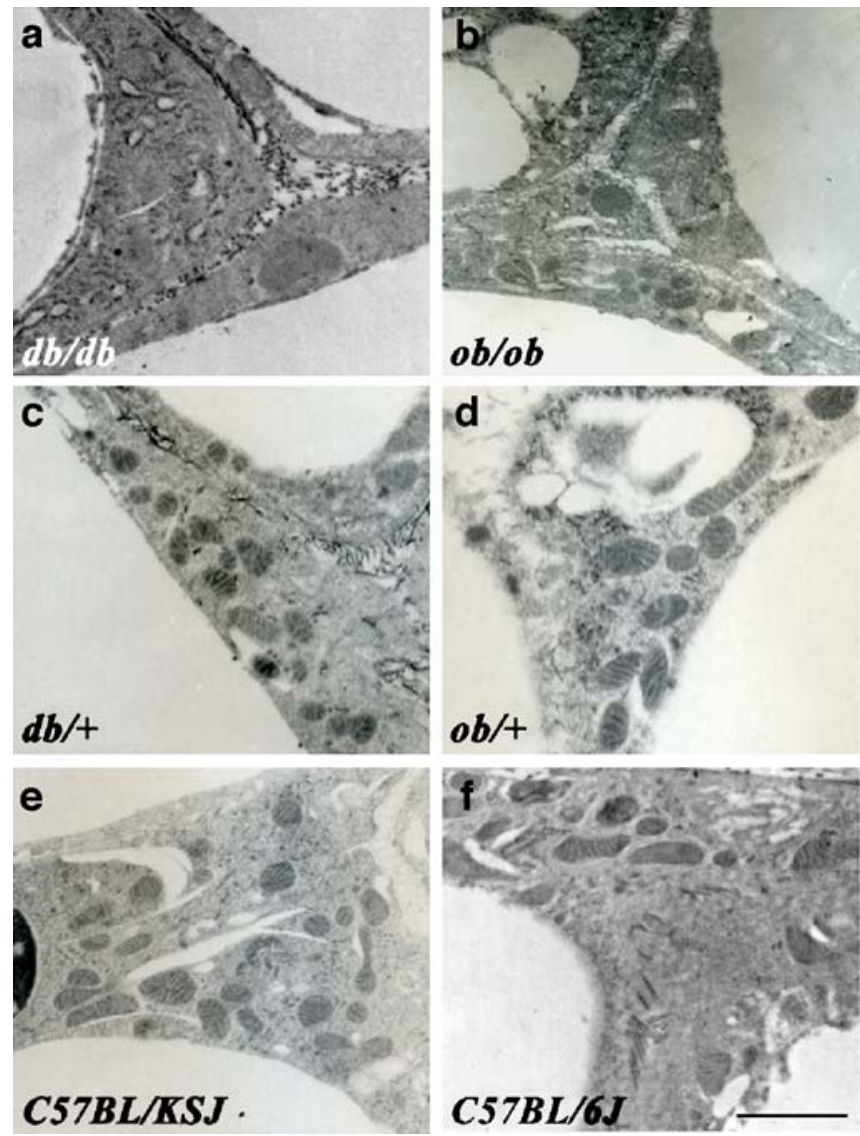

Fig. 4 Mitochondria are rarely found in adipocytes from $d b / d b$ mice under electron microscopy. Epididymal adipose tissues were isolated from 12-week-old male $d b / d b(\mathbf{a}), d b /+(\mathbf{c})$ and $+/+(\mathrm{C} 57 \mathrm{BL} / \mathrm{KSJ})(\mathbf{e})$, and $o b / o b(\mathbf{b}), o b /+(\mathbf{d})$ and $+/+(\mathrm{C} 57 \mathrm{BL} / 6 \mathrm{~J})(\mathbf{f})$ mice. These tissues were fixed, sliced and then observed under electron microscopy. The black bar indicates $1 \mu \mathrm{m}$

the mitochondrial dysfunction in obese and diabetic adipocytes, we determined the respiration rate of adipocytes from normal, ob/ob and $d b / d b$ mice. Fig. $5 b$ shows that $o b / o b$ and $d b / d b$ adipocytes had lower respiration rates than wild-type (C57BL/6J and C57BL/KSJ) adipocytes. From these data, we conclude that $o b / o b$ and $d b / d b$ adipocytes demonstrate similar mitochondrial dysfunction.

Mitochondrial revival in $\mathrm{db} / \mathrm{db}$ adipocytes after rosiglitazone treatment To ascertain whether rosiglitazone alleviates type 2 diabetes by increasing adipocyte mitochondrial biogenesis, 12-week-old $d b / d b$ mice were treated with rosiglitazone $(30 \mathrm{mg} / \mathrm{kg}$ daily) for 10 days. After the treatment, we assessed blood glucose levels, mitochondrial protein levels and morphology. As shown in Fig. 6a, the blood glucose levels were dramatically reduced from 29.3 to $15.2 \mathrm{mmol} / \mathrm{l}$ in $d b / d b$ mice following rosiglitazone treatment, indicating that rosiglitazone is an effective agent for blood glucose lowering and the alleviation of type 2 diabetes. As rosiglitazone induces adipocyte differentiation, fat mass and body weight tend to gradually increase in rosiglitazone-treated mice. Indeed, the body weight of the $d b / d b$ mice was found to have slightly increased after rosiglitazone treatment (Fig. 6b). 
a

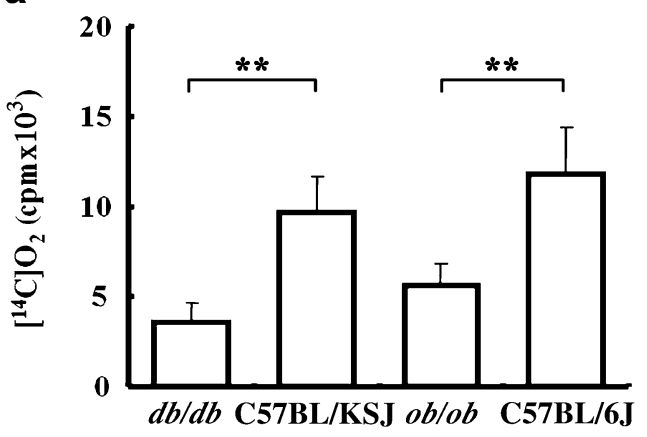

b

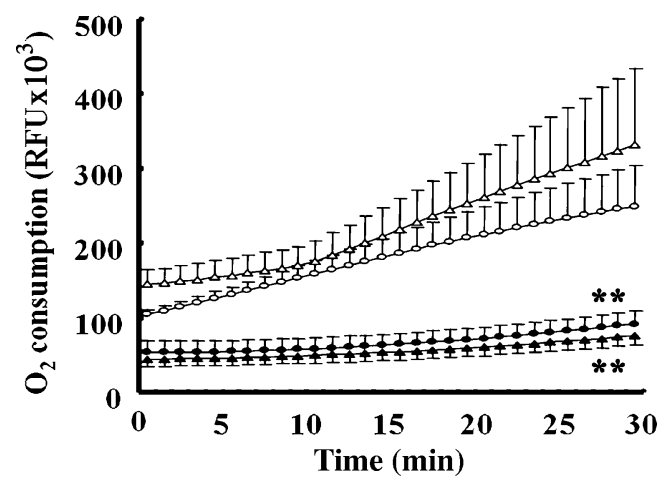

Fig. 5 Mitochondrial malfunction in fatty acid oxidation and respiration in $o b / o b$ and $d b / d b$ adipocytes. Adipocytes were isolated from the epididymal adipose tissues of 12-week-old male $\mathrm{db} / \mathrm{db}, \mathrm{ob} /$ $o b, \mathrm{C} 57 \mathrm{BL} / \mathrm{KSJ}$ and $\mathrm{C} 57 \mathrm{BL} / 6 \mathrm{~J}$ mice. a Fatty acid oxidation of adipocytes. Palmitic acid oxidation was estimated as described in the Materials and Methods section. The number of cells in $100 \mu \mathrm{l}$ of packed volume were estimated by direct DNA measurement. b Oxygen consumption of adipocytes isolated from 12-week-old male db/db (black triangles), ob/ob (black circles), C57BL/KSJ (white triangles) and $\mathrm{C} 57 \mathrm{BL} / 6 \mathrm{~J}$ mice (white circles). Oxygen consumption was measured as described in the Materials and Methods section. Data are presented as means and vertical lines represent the standard deviation of experiments performed in triplicate. The number of cells in $50 \mu \mathrm{l}$ of packed volume was estimated by direct DNA measurement. ${ }^{* *} p<0.01$ vs control. $R F U$ relative fluorescent units

Next, we determined the expression levels of mitochondrial proteins, including ATP synthase $\alpha$ and $\beta$, OxPhos II and OxPhos III, by immunoblotting adipocyte proteins from rosiglitazone-treated $d b / d b$ mice. As expected, there were high levels of mitochondrial proteins in the adipocytes from wild-type mice, but low in $d b / d b$ adipocytes. However, high levels of ATP synthase $\alpha$ and $\beta$, OxPhos II and OxPhos III were induced in the adipocytes from the $d b / d b$ mice after rosiglitazone treatment (Fig. 6c, and ESM Table 2). In contrast to those in adipocytes, mitochondrial protein levels were not affected in the liver or muscle of $d b / d b$ mice after the administration of rosiglitazone.

To confirm mitochondrial biogenesis in the $d b / d b$ adipocytes after rosiglitazone treatment, adipocytes were observed under both light and electron microscopy. Under light microscopy, in the $d b / d b$ adipocytes, a single lipid droplet was so large that little space remained for the cytoplasm (Fig. 7a). Conversely, the adipocytes from rosiglitazone-treated $d b / d b$ mice were characterised by a multiplicity of tiny lipid droplets (Fig. 7b). In addition, electron microscopy revealed that there were only a few mitochondria in the adipocytes from $d b / d b$ mice, whereas there were many in the rosiglitazone-treated $d b / d b$ adipocytes (Fig. 7c,d).

\section{Discussion}

Accumulating evidence strongly suggests that mitochondrial dysfunction is linked to both insulin resistance and type 2 diabetes. The mitochondrial protein ATP synthase $\beta$ is significantly reduced and phosphorylated in the muscle of type 2 diabetic patients compared with the muscle of control subjects [6]. The muscle of type 2 diabetic patients exhibits a $30 \%$ reduction in mitochondrial respiration in comparison with controls when assessed by ${ }^{31} \mathrm{P}$-magnetic resonance spectroscopy [10]. Moreover, biopsy muscle samples from type 2 diabetic patients exhibit a seven-fold reduction in subsarcomlemmal mitochondria electron transport chain activity (determined by measuring succinate oxidase activity), in addition to mitochondrial loss (under electron microscopy), compared with samples from lean subjects [8]. All these data point to mitochondrial dysfunction in type 2 diabetic muscle. However, we could not find any evidence for the mitochondrial dysfunction in the skeletal muscle of $o b / o b$ and $d b / d b$ mice (Fig. 1). Since $d b / d b$ mice are not likely to reflect all phenotypes shown in human type 2 diabetes, there could be a discrepancy between human and $d b / d b$ type 2 diabetes in terms of mitochondrial dysfunction. For example, human type 2 diabetes has clearly multifactorial and multigenetic causes $[1,2]$, whereas type 2 diabetes of $d b / d b$ mice has only one cause, leptin receptor mutation.

Mitochondrial dysfunction in muscles might be intimately connected with type 2 diabetes. However, nobody has yet focused on the role of adipocyte mitochondria in the development of type 2 diabetes. Here, we have demonstrated for the first time that adipocytes in diabetic $(d b / d b)$ mice, an animal model of type 2 diabetes, also exhibit mitochondrial dysfunction. We have demonstrated mitochondrial loss in adipocytes from $d b / d b$ and $o b / o b$ mice by determining cellular levels of mitochondrial proteins via immunoblotting, measuring the mtDNA content of the cell by PCR, MitoTracker staining and observing mitochondria under electron microscopy (Figs 1, 2, 3, 4). Mitochondrial loss in the $d b / d b$ adipocytes might lead to a lack of ATP, thereby attenuating lipid biosynthesis. Thus, $d b / d b$ adipocytes may have a reduced ability to utilise glucose in lipid biosynthesis, thus leading to high blood glucose levels in the animals. Although the adipocytes of obese $(o b / o b)$ mice express mitochondrial proteins at levels similar to the adipocytes of wild-type mice, their mtDNA content is slightly reduced, and the mitochondria in the $o b / o b$ adipocytes are smaller than those in the wild-type adipocytes. This observation suggests that mitochondrial dysfunction might be present in the adipocytes of $o b / o b$ mice. Alternatively, extreme hyperglycaemia (blood glucose level $29 \mathrm{mmol} / \mathrm{l})$ in $d b / d b$ mice could lead to a 


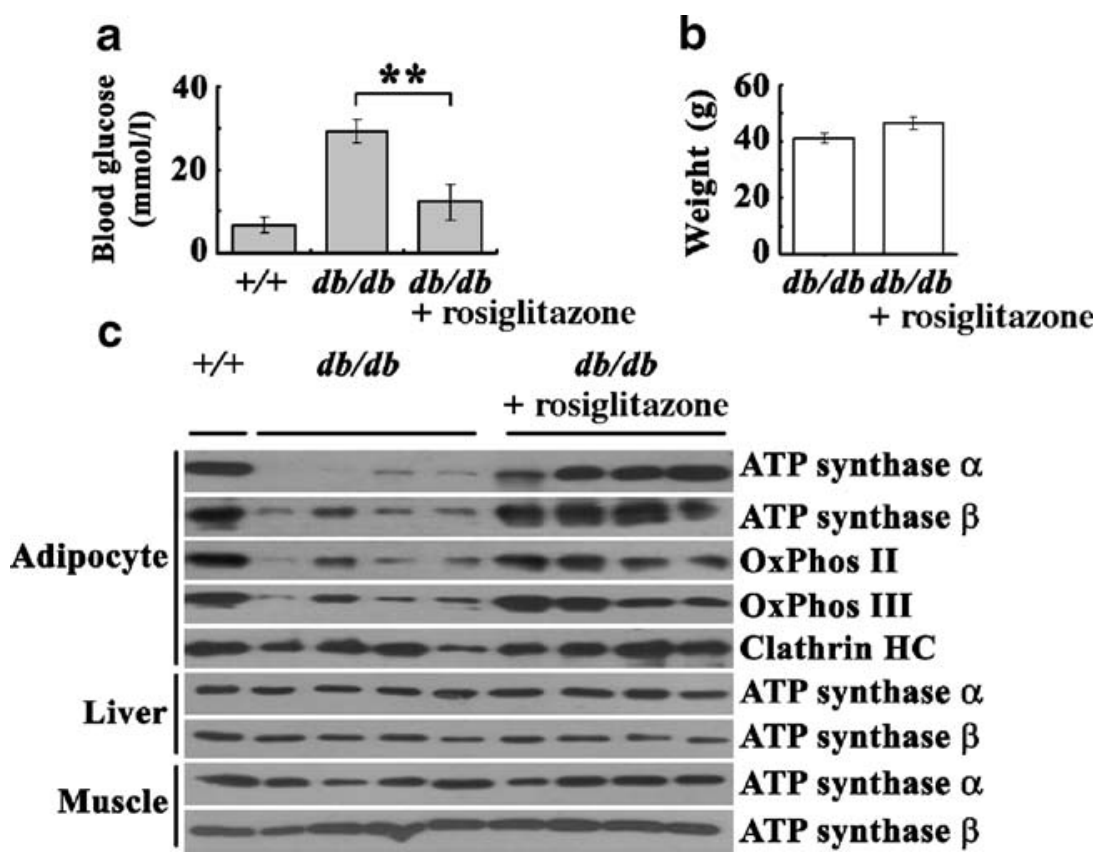

Fig. 6 Mitochondria are increased in adipocytes from $d b / d b$ mice after rosiglitazone treatment. Mice ( 12 weeks old, $d b / d b, n=7$ ) were administered $30 \mathrm{mg} / \mathrm{kg}$ of ground rosiglitazone maleate for 10 days by oral treatment with gavage. Control $d b / d b$ mice $(n=7)$ were treated with the vehicle, $1 \%$ carboxymethyl cellulose. The mice were weighed (b) and bled via the tail vein in order to measure blood glucose (a) with Glucotrend2 (Roche Diagnostics, Vienna, Austria). c.

glycolytic state in the adipocytes, with a concomitant reduction in mitochondrial number and functionality. In addition, low hyperglycaemia (blood glucose level $12 \mathrm{mmol} / \mathrm{l}$ ) in $o b / o b$ mice could be accompanied by

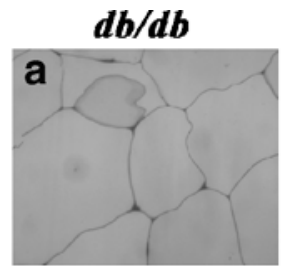

$d b / d b$

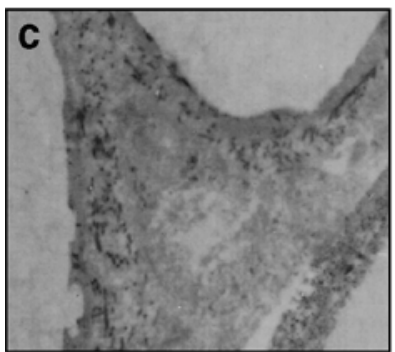

Fig. 7 Mitochondrial biogenesis is induced in the adipocytes of $d b /$ $d b$ mice after rosiglitazone treatment. Rosiglitazone $(30 \mu \mathrm{g} / \mathrm{kg})$ was administered to 12 -week-old $d b / d b$ mice $(n=7)$ by oral treatment with gavage for 10 days. Control $d b / d b$ mice $(n=7)$ were treated with the vehicle, $1 \%$ carboxymethyl cellulose. Epididymal adipose tissue was isolated from control and rosiglitazone-treated $d b / d b$ mice, fixed and then observed under light microscopy $(\mathbf{a}, \mathbf{b})$ and electron microscopy $(\mathbf{c}, \mathbf{d})$. The black bar in $\mathbf{b}$ indicates $2.5 \mu \mathrm{m}$. The black bar in (d) indicates $0.5 \mu \mathrm{m}$
Adipocyte proteins were analysed by immunoblotting with anti-ATP synthase $\alpha$ and $\beta$, anti-OxPhos II (succinate:ubiquinone oxidoreductase $70 \mathrm{kDa}$ subunit), and III (ubiquinone:cytochrome c oxidoreductase core I subunit) and anti-clathrin heavy chain (clathrin $\mathrm{HC}$ ) antibodies. Muscle and liver proteins were analysed by immunoblotting with antibodies directed against ATP synthase $\alpha$ and $\beta$

mitochondrial dysfunction in fatty acid oxidation and mitochondrial respiration (Fig. 5). The effect of rosiglitazone (Fig. 6) could also be explained by an initial decrease in glycaemia followed by normalisation of mitochondrial function.

PPAR $\gamma$ is a nuclear transcription factor that upregulates the expression of adipocytic genes, such as lipoprotein lipase (hydrolysis of lipoproteins) and fatty acid transporter 1 , and lowers levels of NEFA in blood [12]. Thus, PPAR $\gamma$ agonists, most notably rosiglitazone (a member of the thiazolidinedione drug class), reduces lipotoxicity and increases insulin sensitivity in the muscle and liver [12]. In addition, PPAR $\gamma$ agonists inhibit the production of TNF and resistin, both of which promote insulin resistance, but activate the production of adiponectin, which sensitises insulin action in the muscles and liver [13]. PPAR $\gamma$ agonists might also sensitise insulin signalling by stimulating an increase in the number of mitochondria in the adipocytes, as rosiglitazone induces mitochondrial biogenesis in fully differentiated 3T3-L1 adipocytes as well as in the adipocytes of $o b / o b$ mice $[18,20]$. Moreover, we detected mitochondrial biogenesis in the adipocytes of rosiglitazone-treated $d b / d b$ mice (Figs. 6,7 ). As rosiglitazone induces fatty acid oxidation in the adipocytes of $o b / o b$ mice [20], mitochondrial biogenesis results in increases in lipogenesis and lipolysis in the adipocytes, causing a clearance of fatty acids from the blood.

Gain-of-function approaches in cultured cells, virally transduced animals or transgenic mice have shown that PPAR $\gamma$ coactivator $1 \alpha(\mathrm{PGC} 1 \alpha)$ is a key regulator of 
mitochondrial biogenesis and respiration, hepatic gluconeogenesis and muscle fibre-type switching [23-25]. However, PGC1 $\alpha$-deficient mice do not exhibit mitochondrial loss in brown adipose tissue under electron microscopy [26], thereby challenging the role of PGC1 $\alpha$ as a key regulator of mitochondrial biogenesis. Our data also demonstrate that neither the number nor levels of mitochondrial proteins are reduced in adipocytes from $o b / o b$ mice, which do not express PGC1 $\alpha$ [27] (Figs 1, 2, 3). All these findings point to an as yet unclear alternative mechanism for mitochondrial biogenesis in adipocytes. As mitochondrial biogenesis in adipocytes appears to mitigate insulin resistance, any drug that induces mitochondrial proliferation would be a good candidate drug for type 2 diabetes as well as for obesity.

Acknowledgement This work was supported by a grant to Y. Ko from the Korea Research Foundation (KRF-2002-070-C00074). The authors have declared that no conflict of interest exists.

\section{References}

1. Moller DE (2001) New drug targets for type 2 diabetes and the metabolic syndrome. Nature 414:821-827

2. Marx J (2002) Unraveling the causes of diabetes. Science 296:686-689

3. Janson J, Laedtke T, Parisi JE, O'Brien P, Petersen RC, Butler PC (2004) Increased risk of type 2 diabetes in Alzheimer disease. Diabetes 53:474-481

4. Schrauwen P, Hesselink MK (2004) Oxidative capacity, lipotoxicity, and mitochondrial damage in type 2 diabetes. Diabetes 53:1412-1417

5. Taylor R (2004) Causation of type 2 diabetes - the Gordian knot unravels. N Engl J Med 350:639-641

6. Hojlund K, Wrzesinski K, Larsen PM et al (2003) Proteome analysis reveals phosphorylation of ATP synthase beta-subunit in human skeletal muscle and proteins with potential roles in type 2 diabetes. J Biol Chem 278:10436-10442

7. Kelley DE, He J, Menshikova EV, Ritov VB (2002) Dysfunction of mitochondria in human skeletal muscle in type 2 diabetes. Diabetes 51:2944-2950

8. Ritov VB, Menshikova EV, He J, Ferrell RE, Goodpaster BH, Kelley DE (2005) Deficiency of subsarcolemmal mitochondria in obesity and type 2 diabetes. Diabetes 54:8-14

9. Petersen KF, Befroy D, Dufour S et al (2003) Mitochondrial dysfunction in the elderly: possible role in insulin resistance. Science 300:1140-1142

10. Petersen KF, Dufour S, Befroy D, Garcia R, Shulman GI (2004) Impaired mitochondrial activity in the insulin-resistant offspring of patients with type 2 diabetes. N Engl J Med 350:664671
11. Abel ED, Peroni O, Kim JK et al (2001) Adipose-selective targeting of the GLUT4 gene impairs insulin action in muscle and liver. Nature 409:729-733

12. Evans RM, Barish GD, Wang Y-X (2004) PPARs and the complex journey to obesity. Nat Med 10:355-361

13. Fasshauer M, Paschke R (2003) Regulation of adipocytokines and insulin resistance. Diabetologia 46:1594-1603

14. Ahima RS, Flier JS (2000) Leptin. Annu Rev Physiol 62:413437

15. Yamauchi T, Kamon J, Minokoshi Y et al (2002) Adiponectin stimulates glucose utilization and fatty-acid oxidation by activating AMP-activated protein kinase. Nat Med 8:12881295

16. Banerjee RR, Rangwala SM, Shapiro JS et al (2004) Regulation of fasted blood glucose by resistin. Science 303:1195-1198

17. Hotamisligil GS, Peraldi P, Budavari A, Ellis R, White MF, Spiegelman BM (1996) IRS-1-mediated inhibition of insulin receptor tyrosine kinase activity in TNF $\alpha$ and obesity-induced insulin resistance. Science 271:665-668

18. Wilson-Fritch L, Burkart A, Bell G et al (2003) Mitochondrial biogenesis and remodeling during adipogenesis and in response to the insulin sensitizer rosiglitazone. Mol Cell Biol 23:1085-1094

19. Kim B-W, Choo H-J, Lee J-W, Kim J-H, Ko Y-G (2004) Extracellular ATP is generated by ATP synthase complex in adipocyte lipid rafts. Exp Mol Med 36:476-485

20. Wilson-Fritch L, Nicoloro S, Chouinard M et al (2004) Mitochondrial remodeling in adipose tissue associated with obesity and treatment with rosiglitazone. J Clin Invest 114:1281-1289

21. Kanemoto N, Hishigaki H, Miyakita A et al (1998) Genetic dissection of "OLETF", a rat model for non-insulin-dependent diabetes mellitus. Mamm Genome 9:419-425

22. Antonicka H, Ogilvie I, Taivassalo T et al (2003) Identification and characterization of a common set of complex I assembly intermediates in mitochondria from patients with complex I deficiency. J Biol Chem 278:43081-43088

23. Wu Z, Puigserver P, Andersson U et al (1999) Mechanisms controlling mitochondrial biogenesis and respiration through the thermogenic coactivator PGC-1. Cell 98:115-124

24. Lehman JJ, Barger PM, Kovacs A, Saffitz JE, Medeiros DM, Kelly DP (2000) Peroxisome proliferator-activated receptor gamma coactivator-1 promotes cardiac mitochondrial biogenesis. J Clin Invest 106:847-856

25. Lin J, Wu H, Tarr PT et al (2002) Transcriptional co-activator PGC-1 alpha drives the formation of slow-twitch muscle fibres. Nature 418:797-801

26. Lin J, W P-H, Tarr PT et al (2004) Defects in adaptive energy metabolism with CNS-linked hyperactivity in PGC-1alpha null mice. Cell 119:121-135

27. Kakuma T, Wang ZW, Pan W, Unger RH, Zhou YT (2000) Role of leptin in peroxisome proliferator-activated receptor gamma coactivator-1 expression. Endocrinology 141:4576-4582 